\title{
Retardo no diagnóstico e no tratamento cirúrgico do câncer de pulmão*
}

\section{Delay in the diagnosis and surgical treatment of lung cancer}

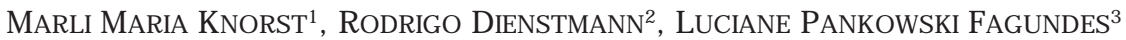

Introdução: 0 câncer de pulmão é a principal causa de morte relacionada a câncer no mundo.

O bjetivo: Determinar o tempo despendido com o diagnóstico, o estadiamento e o tratamento cirúrgico do câncer de pulmão em um hospital universitário.

Método: Entre janeiro de 1990 e dezembro de 1998, 69 pacientes receberam diagnóstico de câncer de pulmão e foram tratados com cirurgia no Hospital das Clínicas de Porto A legre. Os prontuários destes pacientes (53 homens e 16 mulheres) foram revisados, sendo coletados dados clínicos, histológicos, datas de consultas ambulatoriais, de internação hospitalar, de exames e de procedimentos, assim como o local de investigação.

Resultados: Quinze pacientes foram investigados em ambulatório (grupo I), 28 em ambulatório e durante internação (grupo II) e 26 durante internação (grupo III). A idade dos pacientes variou entre 43 e 79 anos (média de $61 \pm 10$ anos). Q uarenta e três pacientes eram tabagistas atuais e 23 ex-tabagistas. 0 tempo médio do aparecimento dos sintomas até a primeira consulta foi de 110 dias e da primeira consulta até o diagnóstico foi de 33 dias. V inte e cinco dias transcorreram entre o diagnóstico e a cirurgia. A média de tempo da primeira consulta até a cirurgia (tempo total) foi de 58 dias. A média do tempo total foi de 72 dias no grupo I, 72 dias no grupo II e 35 dias no grupo III $(p<0,01)$. Não houve diferença significativa no tempo total, considerando-se os diferentes estádios da doença $(p=0,16)$.

Conclusões: Os resultados mostraram retardo na procura de atendimento médico e retardo adicional no tratamento do câncer de pulmão em pacientes cuja investigação foi ambulatorial. (J Pneumol 2003;29(6):358-64)

Descritores - Neoplasias pulmonares/ cirurgia. A vanços da doença.
Background: Lung cancer is the leading cause of cancer-related death worldwide.

O bjective: To determine the time spent diagnosing, staging and surgically treating lung cancer in a university hospital.

Method: Between J anuary 1990 and December 1998, 69 (53 male, 16 female) patients were diagnosed with lung cancer and underwent surgery. The hospital records of these patients were reviewed to evaluate clinical and histological data, dates of outpatient visits, hospital admission, tests and procedures, as well as to determine if the patients were examined as inpatients or as outpatients.

Results: The 15 patients investigated as outpatients were designated as group I, the 28 examined as both inpatients and outpatients as group II, and the 26 investigated as inpatients as group III. A ge ranged from 43 to 79 years (mean \pm SD; $61 \pm 10$ ). Of the 69 patients, 43 were smokers and 23 were ex-smokers. The mean time from onset of symptoms to the first outpatient visit was 110 days and 33 days from the first outpatient visit until diagnosis. There was a lapse of 25 days between diagnosis and surgery. The mean total elapsed time between the first outpatient visit and surgery was 58 days. The mean total time was 72 days for group I, 72 days for group II, and 35 days for group III $(p<0.01)$. There was no significant difference regarding total time in relation to the different stages of the disease $(p=0.16)$.

Conclusions: The results show that patients waited too long before seeking medical assistance and that medical treatment of lung cancer was further delayed when patients were examined in an outpatient setting.

Key words - Lung neoplasm/ surgery. Disease progression.

* Trabalho realizado no Hospital de Clinicas da U niversidade Federal do Rio Grande do Sul, Porto Alegre, RS.

1. Professora Adjunta. Médica Assistente.

2. M édico Residente em Medicina Interna.

3. Médica Residente em Pediatria.

Endereço para correspondência - Marli Knorst, Rua Barros Cassal, 585/ 8
- 90035-030 - Porto A legre, RS, Brasil. Tels.: (51) 3311-4832 e (51)
3333-3739; e-mail: mmknorst@terra.com.br, rdienstmann@cpovo.net

Recebido para publicação em 7/4/03. Aprovado, após revisão, em 13/8/03. 


\section{Veja cometários no Editorial, na página 339}

\section{INTRODUÇÃO}

O câncer de pulmão, neoplasia epitelial primária de pulmão, representa cerca de $90 \%$ de todos os tumores que acometem esse órgão. Segundo estimativa do Ministério da Saúde, 22.085 novos casos de câncer de pulmão deverão ocorrer no Brasil em 2003.(1) Estima-se que, em 2001, o câncer de pulmão tenha causado cerca de um milhão de mortes no mundo e que a sua incidência esteja aumentando em 0,5\% ao ano.(2) Esse tipo de câncer é responsável, já há muitos anos, pela maioria das mortes de causa neoplásica no sexo masculino. Nas últimas décadas, tornou-se a primeira causa de morte por câncer entre as mulheres nos países desenvolvidos, superando os óbitos decorrentes de neoplasia da mama. (3) No Brasil, a principal causa de morte por câncer entre as mulheres ainda é o câncer de mama.(1) Entretanto, o número de óbitos por câncer de pulmão entre as mulheres vem aumentando nos últimos anos. ${ }^{(4)}$

O câncer de pulmão pode ser dividido em dois grandes grupos: carcinoma de pequenas células e carcinoma de células não pequenas. Essa divisão é utilizada por razões terapêuticas e de prognóstico. Entre os principais tipos histológicos no grupo de células não pequenas estão o adenocarcinoma, o carcinoma escamoso ou epidermóide e o carcinoma de grandes células. A ressecção tumoral é considerada o tratamento de eleição nos estádios mais precoces do câncer de pulmão de células não pequenas. Entretanto, na maioria dos casos o diagnóstico é realizado tardiamente, quando os pacientes apresentam sintomas relacionados ao próprio tumor, ou secundários a metástases ou síndromes paraneoplásicas.

É comumente aceito que, no curso de uma neoplasia, tanto o diagnóstico quanto a terapia devam ser instituídos o mais brevemente possível. 0 retardo no tratamento pode ser atribuído ao paciente, que demora a procurar atendimento médico, ou ao sistema de saúde, que não funciona adequadamente. Diversos estudos avaliaram o tempo gasto para investigação e tratamento do câncer de pulmão e detectaram retardo excessivo, tanto relacionado ao paciente $e^{(5-11)}$ quanto ao sistema de saúde no qual o paciente foi atendido.(12-17) Segundo o Standing Medical Advisory Committee da Inglaterra, o tempo de investigação até a ressecção do tumor não deveria ultrapassar seis a oito semanas. ${ }^{(18)}$ Entretanto, o impacto do tempo despendido até o início do tratamento do câncer de pulmão na sobrevida dos pacientes continua sendo discutido. ${ }^{(19,20)}$

0 presente estudo teve por objetivo avaliar o tempo necessário para o diagnóstico e para o tratamento cirúr-
Siglas e abreviaturas utilizadas neste trabal ho

AP - Anatomopatológico

CP - Citopatológico

GI - Grupo I - Pacientes avaliados em ambulatório

GII - Grupo II - Pacientes avaliados em ambulatório e internação hospitalar

GIII - Grupo III - Pacientes avaliados durante internação hospitalar HCPA - Hospital de Clínicas de Porto Alegre

PO - Pneumonia obstrutiva

TC - Tomografia computadorizada

gico do câncer de pulmão em um hospital universitário geral, de acordo com o local de investigação do paciente na instituição: exclusivamente no ambulatório, na internação, ou parte no ambulatório e parte na internação.

\section{MÉTOdo}

Estudo histórico de 69 pacientes submetidos à cirurgia curativa para tratamento de câncer de pulmão no Hospital de Clínicas de Porto Alegre (HCPA), no período de janeiro de 1990 a dezembro de 1998. Todos os pacientes com câncer de pulmão tratados através de cirurgia, atendidos no período acima, foram incluídos no estudo.

Do prontuário médico foram coletados os seguintes dados: idade, sexo, história tabágica, tempo de início dos sintomas, presença de pneumonia obstrutiva (PO), local de investigação, datas de consultas e procedimentos, método que diagnosticou o tumor, exames realizados e tipo histológico do tumor. Todos os pacientes trouxeram ou realizaram radiografia de tórax no início da investigação.

Pneumonia obstrutiva foi diagnosticada pelo médico assistente do paciente na presença de quadro clínico sugestivo, exames laboratoriais e radiografia de tórax compatíveis com infecção.

Para avaliação do tempo despendido para diagnóstico foram consideradas as datas das consultas, dos exames e da cirurgia, sendo admitido o dia um como a data do primeiro atendimento na instituição (consulta ambulatorial ou internação). Foi avaliado o tempo decorrido entre o início dos sintomas e o primeiro atendimento, entre o primeiro atendimento e o diagnóstico e entre o diagnóstico e a cirurgia. Consistiu tempo total o tempo entre o primeiro atendimento e a cirurgia. Os tempos acima foram comparados em dois perío dos seqüenciais de quatro anos e meio, de 1990 a 1994 e de 1994 a 1998. Os pacientes foram divididos em três grupos conforme 0 local de investigação: Gl, com pacientes avaliados no am- 
bulatório; GII, com pacientes avaliados no ambulatório e durante a internação; e GIII, com pacientes investigados somente durante a internação hospitalar.

0 estadiamento tumor-nódulo-metástase (TNM) para cada paciente foi realizado com base nos exames préoperatórios, achados transoperatórios e dados histopatológicos pós-operatórios. 0 estadiamento foi feito de acordo com a rotina do serviço. Radiografia de tórax, fibrobroncoscopia, espirometria, tomografia linear ou computadorizada (TC) de tórax, ecografia ou TC abdominal e cintilografia óssea eram exames recomendados em todos os pacientes. TC de crânio e mediastinoscopia foram realizadas com indicações específicas. Todos os casos foram classificados de acordo com a revisão do estadiamento do câncer de pulmão de 1997.(21)

As informações coletadas foram transferidas para um banco de dados (Epi-Info 6.0) e submetidas à análise estatística. Para comparar o tempo despendido entre o primeiro atendimento e a cirurgia, de acordo com o local de investigação dos pacientes (GI, GII e GIII) e de acordo com o estádio cirúrgico da doença, foi utilizado o teste de KruskalWallis. A nálise posterior foi feita separando-se os pacientes com pneumonia obstrutiva ( $\mathrm{PO}$ ) daqueles que não apresentavam esse diagnóstico no momento da investigação do tumor, uma vez que o mesmo poderia ser um fator de retardo no tratamento dos pacientes. 0 teste de MannWhitney foi utilizado para avaliar o tempo total do início dos sintomas até a cirurgia nos dois períodos seqüenciais de tempo, o tempo da internação até a cirurgia e o tempo total em pacientes investigados em ambulatório ou internação, com e sem Po. Os dados foram expressos através de freqüência simples e percentagem, média e desvio padrão (DP) ou mediana e intervalo de confiança. U m valor de $p<0,05$ foi estabelecido para significância estatística.

O estudo foi aprovado pelo Comitê de Ética em Pesquisa do Grupo de Pesquisa e Pós-Graduação do HCPA.

\section{Resultados}

Sessenta e nove pacientes foram submetidos à cirurgia por câncer de pulmão no período de janeiro de 1990 a dezembro de 1998, no HCPA. 0 grupo de estudo compreendia 53 homens e 16 mulheres, com média de idade de 61,4 7,1 anos (mediana de 62 anos). Em 15 pacientes o local de investigação foi o ambulatório (GI); em 28 pacientes, o ambulatório e a internação (GII); e 26 pacientes foram investigados durante a internação hospita$\operatorname{lar}(\mathrm{GIII})$.

A história tabágica foi positiva em 66 pacientes (96\%). Em 51 prontuários estava documentado o tempo decorrido entre o início dos sintomas e a primeira consulta. A média do tempo entre 0 início dos sintomas e a primeira consulta foi de $110,0 \pm 72,5$ dias. Dez pacientes procuraram serviço médico especializado em até 30 dias, nove pacientes entre 30 e 60 dias, 11 pacientes procuraram atendimento médico no período entre 60 e 120 dias do início dos sintomas e os demais, após 120 dias.

0 retardo no diagnóstico em cada etapa da investigação é mostrado na Tabela 1 , através da mediana e da amplitude interquartil do tempo despendido para a realização dos exames. 0 método diagnóstico em $42 \%$ dos pacientes foi o exame anatomopatológico (AP) pós-cirúrgico e, em 40,6\%, o AP ou citopatológico (CP) do material obtido através de fibrobroncoscopia (Tabela 2). 0 tipo histológico encontrado foi adenocarcinoma em 38 pacientes (55\%), escamoso em 26 (38\%), adenoescamoso em um, pequenas células em três pacientes (estes com diagnóstico pós-operatório) e grandes células em um paciente. Quanto ao estádio tumoral, 27 pacientes (39\%) apresentavam estádio I; 18 (26\%), estádio II; e 24 pa-

TABELA 1

Retardo na investigação de 69 pacientes com câncer de pulmão tratados com cirurgia

\begin{tabular}{lccc}
\hline \multicolumn{1}{c}{ Exame } & $\begin{array}{c}\text { Número de } \\
\text { pacientes }\end{array}$ & $\begin{array}{c}\text { Mediana } \\
\text { (dias) }\end{array}$ & $\begin{array}{c}\text { Amplitude } \\
\mathbf{2 5 - 7 5 \%} \text { (dias) }\end{array}$ \\
\hline Fibrobroncoscopia & 54 & 8 & $3-23$ \\
CP escarro & 27 & 9 & $4-31$ \\
Espirometria & 67 & 12 & $7-29$ \\
Cintilografia óssea & 63 & 17 & $5-42$ \\
TC crânio & 29 & 17 & $11-30$ \\
Ecografia abdominal & 46 & 18 & $6-35$ \\
TC tórax & 48 & 22 & $10-41$ \\
M ediastinoscopia & 33 & 32 & $20-68$ \\
\hline
\end{tabular}

$\mathrm{CP}=$ exame citopatológico; $\mathrm{TC}=$ tomografia computadorizada.

TABELA 2

Métodos que forneceram o diagnóstico* em 69 pacientes com câncer de pulmão tratados com cirurgia

\begin{tabular}{lcc}
\hline \multicolumn{1}{c}{ Método } & $\begin{array}{c}\text { N úmero de } \\
\text { pacientes }\end{array}$ & $\begin{array}{c}\text { Percentagem } \\
(\%)\end{array}$ \\
\hline AP pós-cirúrgico & 29 & 42,0 \\
\hline Fibrobroncoscopia & 19 & 27,6 \\
AP de biópsia & 9 & 13,0 \\
CP de lavado ou escovado & 8 & 11,6 \\
\hline CP de escarro & 2 & 2,9 \\
\hline AP de mediastinoscopia & 2 & 2,9 \\
\hline Biópsia pulmonar transcutânea & 2 & \\
\hline * Primeiro exame positivo; AP= exame anatomopatológico; $C P=$ exame citopatológico.
\end{tabular}


cientes (35\%), estádio III. 0 tipo histológico e o estádio dos casos estão detalhados na Tabela 3.

0 tempo despendido entre a primeira consulta e o diagnóstico foi em média de 33,4 \pm 40,2 dias (mediana de 18 dias). Esse tempo foi inferior a 15 dias em 31 pacientes $(45 \%)$ e variou entre 16 e 30 dias em outros 15 pacientes (22\%). Nos demais casos (33\%), o diagnóstico foi retardado em mais de 30 dias (Tabela 4).

TABELA 3

Tipo histológico e estádio do câncer de pulmão em 69 pacientes tratados com cirurgia

\begin{tabular}{lcl}
\hline Tipo histológico & $\begin{array}{c}\text { Número de } \\
\text { pacientes }\end{array}$ & Estádio \\
\hline Escamoso & 1 & IA \\
& 11 & IB \\
& 5 & IIB \\
& 6 & IIIA \\
& 3 & IIIB \\
\hline Adenocarcinoma & 5 & IA \\
& 9 & IB \\
& 1 & IIA \\
& 12 & IIB \\
\hline Adenoescamoso & 11 & IIIA \\
\hline Pequenas células & 1 & IIIA \\
\hline Grandes células & 1 & IA \\
\hline
\end{tabular}

0 tempo gasto entre o diagnóstico e a cirurgia foi em média de 25,0 $\pm 30,8$ dias, com mediana de 20 dias (Tabela 5). Em 29 pacientes (42\%) o diagnóstico foi feito através de material obtido no procedimento cirúrgico. Vinte e dois pacientes (32\%) realizaram a cirurgia nos 30 dias seguintes ao diagnóstico e os demais 18 pacientes (26\%), após 31 dias.

0 tempo total da primeira consulta até cirurgia foi em média de 58,2 $\pm 44,7$ dias, variando de acordo com o local de investigação do paciente (Tabela 5 ). No GI, a média foi de 72,4 $\pm 31,3$ dias (mediana de 63 dias), no GII foi de $72,3 \pm 57,4$ dias (mediana de 53 dias) e no GIII foi de $34,9 \pm 20,7$ dias (mediana de 30 dias), significativamente menor que nos outros grupos $(p<0,01)$. A média do tempo total da primeira consulta até cirurgia não diferiu nos pacientes investigados nos primeiros quatro anos e

TABELA 4

Tempo decorrido entre a primeira consulta e a confirmação diagnóstica em 69 pacientes com câncer de pulmão tratados com cirurgia

\begin{tabular}{|c|c|c|}
\hline Período & $\begin{array}{c}\text { Número de } \\
\text { pacientes }\end{array}$ & $\begin{array}{c}\text { Percentagem } \\
(\%)\end{array}$ \\
\hline 10-150 dia & 31 & 45 \\
\hline $160-30 \div \mathrm{dia}$ & 15 & 22 \\
\hline $310-60$ o dia & 9 & 13 \\
\hline $610-900$ dia & 9 & 13 \\
\hline Acima de 90 dias & 5 & 7 \\
\hline Total & 69 & 100 \\
\hline
\end{tabular}

TABELA 5

Retardo no diagnóstico e no tratamento cirúrgico do câncer de pulmão em 69 pacientes tratados com cirurgia

\begin{tabular}{lccc}
\hline \multirow{2}{*}{ Intervalo de tempo } & \multicolumn{3}{c}{ Tempo despendido (dias) } \\
\cline { 2 - 4 } & Média & Mediana & Amplitude 25-75\% \\
\hline Início dos sintomas até 1ạ consulta & 110 & 90 & $60-180$ \\
\hline 1a consul ta até diagnóstico & 33 & 18 & $7-57$ \\
\hline Diagnóstico até cirurgia & 25 & 20 & $0-36$ \\
\hline 1a consul ta até cirurgia & & & \\
G rupo I & 72 & 63 & $42-93$ \\
G rupo II & 72 & 53 & $30-89$ \\
G rupo III & 35 & 30 & $20-43$ \\
Estádio I & 54 & 39 & $42-74$ \\
Estádio II & 71 & 63 & $29-65$ \\
Estádio III & 53 & 35 & \\
\hline
\end{tabular}

GI - investigação ambulatorial; GII - investigação ambulatorial e durante internação hospitalar; GIII - investigação durante internação hospitalar. 
meio (32 pacientes, $57,8 \pm 45,9$ dias) e nos últimos quatro anos e meio ( 37 pacientes, $58,7 \pm 44,3$ dias; $p=$ $0,87)$. Não houve diferença significativa no tempo total quando comparados os diferentes estádios da doença ( $p$ $=0,16)$. Também não houve diferença significativa no estádio da doença quando comparados os três grupos de pacientes de acordo com o local de investigação $(p>0,05)$.

Dezoito pacientes $(26 \%)$ apresentaram Po, sete deles investigados em ambulatório e internação e 11 investigados durante internação. A média do tempo total da primeira consulta até a cirurgia nos pacientes com Po foi de $51,4 \pm 23,3$ dias e, sem PO $(n=51)$, de 60,6 \pm 50,2 dias $(p=0,79)$. Entretanto, a média do tempo de internação até a cirurgia foi significativamente menor no grupo de pacientes sem PO (16,8 $\pm 15,7$ dias), quando comparado com o grupo com PO $(34,8 \pm 21,1$ dias) $(p<0,01)$. Q uando analisados os dados dos pacientes sem PO, a média do tempo total da primeira consulta até cirurgia foi de 26,0 $\pm 16,0$ dias nos pacientes investigados em internação ( $\mathrm{n}$ $=15$ ), e de 75,0 $\pm 52,6$ dias nos pacientes que foram parcial ou totalmente investigados em ambulatório ( $\mathrm{n}=$ 36; $p<0,01)$.

\section{DISCUSSÃo}

A ressecção cirúrgica continua sendo o tratamento de escolha para o câncer de pulmão de células não pequenas nas fases iniciais da doença. Entretanto, a realização da cirurgia está na dependência da operabilidade do paciente e da ressecabilidade do tumor. A ressecabilidade da lesão depende de diagnóstico e de intervenção precoces, enquanto o tumor permanece localizado. No câncer de pulmão de células não pequenas estádio I, a sobrevida em cinco anos é de 70 a $80 \%$, (22) enquanto que nos estádios mais avançados (III e IV) a mortalidade no mesmo período é superior a $90 \%$. 23,24$)$

0 presente estudo evidenciou que 0 acesso ao hospital para investigação dos sintomas relacionados ao câncer de pulmão é, em geral, demorado. Procuraram assistência médica após 60 dias do início dos sintomas $58 \%$ dos pacientes. Recentemente Lee et al., ${ }^{\left({ }^{9}\right)}$ numa série de 90 pacientes ingleses, descreveram retardo de 39 dias do início dos sintomas até a realização da primeira radiografia de tórax e de 112 dias, entre o início dos sintomas e o encaminhamento do paciente para o cirurgião. Mor et al. ${ }^{(10)}$ observaram retardo de mais de três meses na procura por assistência médica em $25 \%$ e Hackett et al., ${ }^{(7)}$ em $39 \%$ dos pacientes. Um tempo prolongado entre 0 início dos sintomas e a consulta médica, variando de 2,4 a 10,8 meses, foi igualmente relatado por outros autores. $(5,6,8,11)$ Contrastando com os dados acima, o estudo com 300 pacientes realizado por Silva et al.,(17) entre 1988 e 1991, mostrou que a maioria dos pacientes $(69,6 \%)$ procurou assistência médica em até 30 dias após o início clínico da doença. Dados semelhantes foram observados em 134 pacientes suíços, que procuraram atendimento médico após 43 dias (mediana de 21 dias) do início dos sintomas. ${ }^{(25)} \mathrm{O}$ intervalo mais curto entre início dos sintomas e consulta médica (mediana de sete dias) foi relatado na Itália. (26)

Em relação à cronologia e à freqüência da realização dos exames, observamos que a fibrobroncoscopia foi 0 procedimento mais precocemente realizado (mediana de oito dias). Esses dados são semelhantes aos da casuística de Lopez Encuentra et al., (27) na qual os pacientes realizaram broncoscopia em média após 9,5 dias do primeiro atendimento, com um tempo mais curto, de 3,1 dias, no grupo de pacientes internados. $\mathrm{Na}$ nossa série, apenas $39,1 \%$ dos pacientes realizaram exame CP de escarro. 0 exame CP de escarro é um exame não invasivo cuja positividade depende do tamanho e localização do tumor. Em tumores grandes e centrais, a positividade de múltiplas amostras pode alcançar $85 \% .{ }^{(28)}$ Adicionalmente, a tomografia computadorizada (TC) de tórax, cujos dados poderiam contribuir durante a realização da fibrobroncoscopia, em muitos casos foi realizada posteriormente (mediana de 22 dias). A demora esperada para realização do exame CP do escarro e da TC de tórax na nossa instituição e a facilidade da realização da broncoscopia pelo pneumologista, associadas ao fato da broncoscopia fazer parte do estadiamento dos casos de câncer de pulmão potencialmente cirúrgicos, podem estar relacionadas à realização mais precoce deste último exame.

O tempo gasto entre a primeira consulta e a confirmação da neoplasia ultrapassou 30 dias em um terço de nossos pacientes, sendo a média de 33 dias. Na série de Silva et al. ${ }^{(17)}$ esse tempo foi superior a 90 dias em $56 \%$ dos pacientes, representando retardo ainda maior para 0 diagnóstico. Para a G rupo Italiano Interdisciplinar de A valiação do Câncer,(26) 0 tempo gasto entre o primeiro sintoma e o diagnóstico final foi, em média, de 50 dias. Um dos fatores que pode ter contribuído decisivamente para aumentar o tempo entre o primeiro atendimento e o diagnóstico de câncer de pulmão, em nosso hospital, é o fato de que, em $42 \%$ dos casos, o diagnóstico foi obtido através do exame AP pós-cirúrgico, ou seja, toda a rotina de investigação realizada previamente havia sido negativa.

Neste estudo, a média do tempo despendido entre 0 diagnóstico e a cirurgia (atraso terapêutico) foi de 25 dias e mais de $70 \%$ dos pacientes foram operados no período de 30 dias após o diagnóstico. Esses dados coincidem com os do estudo de Ringbaek et al., ${ }^{(14)}$ que demonstraram atraso terapêutico em média de 26 dias, sendo que $95 \%$ dos 83 pacientes com câncer de pulmão ressecável foram operados em 60 dias. Billing e Wells, ${ }^{(12)}$ que avaliaram 38 pacientes com câncer de pulmão atendidos em 
um hospital da Inglaterra, observaram um intervalo médio entre a referência para tratamento cirúrgico e a ressecção pulmonar de 24 dias. Outros autores relataram média de 45 e 54 dias em dois grupos de pacientes na Espanha. (27)

Nosso estudo mostrou que pacientes investigados durante internação hospitalar realizaram a ressecção tumoral, em média, na metade do tempo daqueles investigados total ou parcialmente em ambulatório (35 versus 72 dias). Esses resultados sugerem que a investigação ambulatorial é um dos fatores de retardo para o tratamento cirúrgico do câncer de pulmão. Além disso, quando excluídos os pacientes com PO, um possível fator de confusão para a análise do tempo total da primeira consulta até a cirurgia, a média do tempo total foi de 26 dias nos pacientes internados e de 75 dias nos ambulatoriais. 0 tempo entre a primeira consulta médica e a ressecção do câncer de pulmão nos casos de ressecabilidade, entretanto, não deveria ser superior a oito semanas. ${ }^{(18)}$

o tempo de retardo neste estudo não se associou com o estádio do tumor no momento da cirurgia. Resultado semelhante foi referido por outros autores, que não encontraram associação entre retardo no diagnóstico ou tratamento e estádio tumoral ou sobrevida dos pacientes. ${ }^{(10,12,15,29)}$ Contrastando com esses dados, Christensen et al.,(13) em um estudo dinamarquês incluindo 172 pacientes com câncer de pulmão, relataram ser o tempo

\section{REFERÊNCIAS}

1. Brasil. Ministério da Saúde. Estimativa da incidência e mortalidade por câncer no Brasil 2003. Rio de J aneiro: INCA; 2003.

2. Spiro SG, Porte J C. Lung cancer - Where are we today? Current advances in staging and nonsurgical treatment. Am J Respir Crit Care Med 2002;166:1166-96

3. Wingo PA, Ries LAG, Giovino GA, Miller DS, Rosenberg HM, Shopland $D R$, et al. Annual report to the nation on the status of cancer, 1973-1996, with a special section on lung cancer and tobacco smoking. J Natl Cancer Inst 1999;91:675-90.

4. Greenlee RT, Murray T, Balden S, Wingo PA. Cancer statistics 2000. CA Cancer J Clin 2000;50:7-33.

5. Antkowiak J G, Regal AM, Takita H. Bronchogenic carcinoma in patients under age 40. Ann Thorac Surg 1989;47:391-3.

6. De Caro L, Benfield J R. Lung cancer in young persons. J Thorac Surg 1982;83:372-6.

7. Hackett TP, Cassem NH, Raker J W. Patient delay in cancer. N Engl J Med 1973;289:14-20.

8. Kyriakos M, Webber B. Cancer of the lung in young man. J Thorac Cardiovasc Surg 1974;67:634-48.

9. Lee J, Marchbank A, Goldstraw P. Implementation of the British Thoracic Society recommendations for organizing the care of patients with lung cancer: the surgeon's perspective. Ann R Coll Surg Engl 2002; 84:304-8.

10. Mor V, Masterson-Allen S, Goldberg R, Guadagnoli E, Wool MS. Pre-diagnostic symptom recognition and help seeking among cancer patients. J Community Health 1990;15:253-66.

11. Pemberton J N, Nagorney DM, Gilmore J C, Taylor WF, Bernatz PE. Bronchogenic carcinoma in patients younger than 40 years. Ann Thorac Surg 1983;36:509-15 entre o aparecimento dos sintomas e a cirurgia, e entre 0 primeiro contato com o sistema de saúde e a cirurgia menor nos pacientes com melhor prognóstico (estádios I e II). Em outro estudo, seis de 29 pacientes tornaram-se clinicamente incuráveis enquanto aguardavam radioterapia. ${ }^{(16)}$

A demora excessiva para diagnosticar e estadiar a doença contribui para aumentar a angústia do paciente, o que por si só justifica que se avaliem o processo diagnóstico e o tempo necessário para a instituição terapêutica. Diversos fatores podem estar relacionados à ineficiência no atendimento ambulatorial de pacientes com câncer de pulmão no nosso meio: difícil acesso a atendimento médico especializado, problemas na referência e contra-referência de pacientes no sistema de saúde, sobrecarga dos serviços capacitados para diagnosticar e tratar câncer, e falta de uma rotina assistencial específica para a doença. Portanto, se por um lado é necessário identificar precocemente os pacientes em maior risco de desenvolver câncer de pulmão, por outro, é preciso reestruturar 0 atendimento ambulatorial no sistema público de saúde, de forma a racionalizar e agilizar a marcação de consultas e os pedidos de exames com finalidade diagnóstica, reduzindose, assim, o prazo para detecção do câncer.

Em conclusão, nossos dados sugerem que há um retardo significativo na procura por atendimento médico e na investigação ambulatorial do câncer de pulmão.

12. Billing J S, Wells FC. Delays in the diagnosis and surgical treatment of lung cancer. Thorax 1996;51:903-6.

13. Christensen ED, Harvald T, J endresen M, A ggestrup S, Petterson G. The impact of delayed diagnosis of lung cancer on the stage at time of operation. Eur J Cardiothorac Surg 1997;12:880-4.

14. Ringbaek TJ, Borgeskov S, Lange P, Viskum K. Therapeutic and prognostic course in patients with suspected lung cancer. Results of combined thoracic surgery-pulmonary medicine evaluation. U geskr Laeger 1998;160:1705.

15. Robinson E, Mohilever J, Zidan J, Sapir D. Delay in diagnosis of cancer. Possible effects on the stage of disease and survival. Cancer 1984;54:145460 .

16. O'Rourke N, Edwards R. Lung cancer treatment waiting times and tumour growth. Clin Oncol 2000;12:141-4.

17. Silva PA, Pereira J R, Ifari FK, Minamoto H. Câncer de pulmão e retardo no diagnóstico: análise de 300 casos. Rev Assoc Med Bras 1992; 38:145-9.

18. Standing Medical Advisory Committee. Management of lung cancer: current clinical practices. London: Department of Health, 1994.

19. Bozcuk H, Martin C. Does the treatment delay affect survival in non-small cell lung cancer? A retrospective analysis from a single UK centre. Lung Cancer 2001;34:243-52

20. Aragoneses FG, Moreno N, Leon P, Fontan EG, Folque E; Bronchogenic Carcinoma Cooperative Group of the Spanish Society of Pneumology and Thoracic Surgery (GCCB-S). Influence of delays on survival in the surgical treatment of bronchogenic carcinoma. Lung Cancer 2002;36:59-63.

21. Mountain $\mathrm{Cl}$. Revisions in the International System for Staging Lung Cancer. Chest 1997;111:1710-7 
22. Naruke T, Tsuchiya $\mathrm{R}$, Kondo $\mathrm{H}$, A samura $\mathrm{H}$. Prognosis and survival after resection for bronchogenic carcinoma based on the 1997 TNM-staging classification: the J apanese experience. Ann Thorac Surg 2001;71:175964.

23. Jassem J, Skokowski J, Dziadziuszko R, Jassem E, Szymanowska A, Rzyman $W$, et al. Results of surgical treatment of non-small cell lung cancer: validation of the new postoperative pathologic TNM classification. J Thorac Cardiovasc Surg 2000;119:1141-6.

24. Van Rens MT, de la Riviere AB, Elbers HR, van Den Bosch J M. Prognostic assessment of 2,361 patients who underwent pulmonary resection for nonsmall cell lung cancer, Stage I, II, and IIIA. Chest 2000;117:374-9.

25. Koyi H, Hillerdal G, Branden E. Patient's and doctors' delay in the diagnosis of chest tumors. Lung Cancer 2002;35:53-7.
26. G.I.V.I.O. (Interdisciplinary Group for Cancer Evaluation). Diagnosis and first-line treatment of patients with lung cancer in Italian general hospitals. Tumori 1989;75:163-7.

27. Lopez Encuentra A, Martin de Nicolas J L, Casado Lopez M, de Miguel Poch $\mathrm{E}$, Marron Fernandez C. Delays in the diagnosis and surgical treatment of bronchogenic carcinoma. Cooperative Group on Bronchogenic Carcinoma of SEPAR (GCCB-S). Arch Bronconeumol 1998; 34:123-6.

28. Bocking A, Biesterfeld S, Chatelain R, Gien-Gerlach G, Esser E. Diagnosis of bronchial carcinoma on sections of paraffin-embedded sputum. Sensitivity and specificity of an alternative to routine cytology. Acta Cytol 1992;36:37-47.

29. Porta M, Gallen M, Malats N, Planas I . Influence of diagnostic delay upon cancer survival: an analysis of five tumour sites. J Community $\mathrm{Health}$ 1991;45:225-30. 